\title{
Build and Evaluate a Free Virtual Cluster on Amazon Elastic Compute Cloud for Scientific Computing
}

\author{
https://doi.org/10.3991/ijoe.v13i08.7373 \\ Jie Xiong $(\bowtie)$ \\ Yangtze University, Jingzhou, China; \\ Mississippi State University, Starkvill, MS, USA \\ xiongjiedyangtzeu.edu.cn \\ Shen-Han Shi \\ Yangtze University, Jingzhou, China \\ Song Zhang \\ Mississippi State University, Starkvill, MS, USA
}

\begin{abstract}
Scientific computing requires a huge amount of computing resources, but not all the scientific researchers have an access to sufficient highend computing systems. Currently, Amazon provides a free tier account for cloud computing which could be used to build a virtual cluster. In order to investigate whether it is suitable for scientific computing, we first describe how to build a free virtual cluster using StarCluster on Amazon Elastic Compute Cloud (EC2). Then, we perform a comprehensive performance evaluation of the virtual cluster built before. The results show that a free virtual cluster is easily built on Amazon EC2 and it is suitable for the basic scientific computing. It is especially valuable for scientific researchers, who do not have any HPC or cluster, to develop and test their prototype system of scientific computing without paying anything, and move it to a higher performance virtual cluster when necessary by choosing more powerful instance on Amazon EC2.
\end{abstract}

Keywords-Virtual cluster, Amazon Elastic Compute Cloud (EC2), Evaluation, Scientific computing, StarCluster, High Performance Computing (HPC)

\section{Introduction}

Scientific computing is the key to solve the great challenges in many domains and has provided advances in diverse fields of science [1]. Scientific computing has been dependent on High Performance Computing (HPC) and parallel processing, since running large simulation requires a huge amount of computing resources. However, not all scientific researchers have an access to sufficient high-end computing systems [2]. Moreover, some scientific researchers in developing country even do not have any cluster at all.

Cloud computing paradigm proposes the integration of different technological models to provide hardware infrastructure, development platforms, and applications 
as on demand services based on a pay-as-you-go model [3]. Some academic and commercial HPC users are looking at clouds as a cost effective alternative to dedicated HPC clusters [1]. Renting rather than owning a cluster avoids the up-front and operating expenses associated with a dedicated infrastructure [4]. As one of the cloud computing vendors, Amazon provides a web service named Elastic Compute Cloud (EC2) [5] which could be used to build a virtual cloud cluster [6][7][8]. Currently, Amazon provides a free tier account for cloud computing, allowing users to use for free in 12 month. It is a good news for the scientific researchers who need HPC or cluster but do not have it.

Creating a cluster on Amazon EC2 without a tool is somewhat tedious. StarCluster [9], developed by the Massachusetts Institute of Technology, is a powerful toolkit to automate and simplify the process of building, configuring, and managing clusters of virtual machines on Amazon EC2 cloud. We can easily create a cluster computing environment on the Amazon cloud for scientific computing using StarCluster.

Since the Amazon EC2 could be used to build a virtual cluster for free, it is important to answer the follow question: Is is suitable for scientific computing? This paper is dedicated to build a free tier virtual cluster on Amazon cloud with StarCluster, and evaluate its performance for the scientific computing.

\section{Building a free tier virtual cluster on Amazon EC2}

\subsection{Amazon EC2 Instance Types}

Amazon EC2 is a web services which provides resizable compute capability in the Amazon cloud. It provides a wide selection of instance types optimized to fit different use cases. Instance types comprise varying combinations of CPU, memory, storage, and networking capacity and give user the flexibility to choose the appropriate mix of resources for your applications. Each instance type includes one or more instance sizes, allowing user to scale the resources to the requirements of your target workload. The current configuration and price [10] of some typical instance types are listed in Table 1.

For the free tier accounting user, it is free to use 750 hours per month for the t2. micro instance within first 12 months since signing up. According to the policy of Amazon, scientific researchers can build an 8 t2.micro instance (nodes) virtual cluster, and use it 93.75 hours per month for free. It is useful to them to develop their prototype system of scientific computing without paying anything, and move to the higher performance cluster ater when necessary by choosing more powerful instance type listed in Table 1 on Amazon EC2. 
Table 1. Configuration and price of several typical instance types

\begin{tabular}{|c|c|c|c|c|c|c|c|}
\hline Group & $\begin{array}{c}\text { Instance } \\
\text { Type }\end{array}$ & vCPU & $\begin{array}{c}\text { Memory } \\
\text { (GB) }\end{array}$ & Network & $\begin{array}{l}\text { Physical } \\
\text { Processor }\end{array}$ & $\begin{array}{l}\text { Clock } \\
\text { Speed } \\
(\mathbf{G H z}) \\
\end{array}$ & $\begin{array}{c}\text { Price (\$ per } \\
\text { Hour) }\end{array}$ \\
\hline \multirow[t]{3}{*}{$\begin{array}{l}\text { General } \\
\text { Purpose }\end{array}$} & t2.nano & 1 & 0.5 & Low & $\begin{array}{l}\text { Intel Xeon } \\
\text { family }\end{array}$ & up to 3.30 & 0.059 \\
\hline & t2.micro & 1 & 1 & Low to Mod. & $\begin{array}{l}\text { Intel Xeon } \\
\text { family }\end{array}$ & Up to 3.3 & 0.012 \\
\hline & t2.large & 2 & 8 & Low to Mod. & $\begin{array}{l}\text { Intel Xeon } \\
\text { family }\end{array}$ & Up to 3.3 & 0.094 \\
\hline \multirow[t]{3}{*}{$\begin{array}{l}\text { Comput. } \\
\text { Optimized }\end{array}$} & c4.large & 2 & 3.75 & Moderate & $\begin{array}{l}\text { Intel Xeon E5- } \\
2666\end{array}$ & 2.9 & 0.1 \\
\hline & c4.xlarge & 4 & 7.5 & High & $\begin{array}{l}\text { Intel Xeon E5- } \\
2666 \\
\end{array}$ & 2.9 & 0.199 \\
\hline & c4.8xlarge & 36 & 60 & $10 \mathrm{~Gb}$ & $\begin{array}{l}\text { Intel Xeon E5- } \\
2666\end{array}$ & 2.9 & 1.591 \\
\hline \multirow[t]{2}{*}{$\begin{array}{l}\text { GPU In- } \\
\text { stance }\end{array}$} & p2.xlarge & 4 & 61 & High & $\begin{array}{l}\text { Intel Xeon E5- } \\
2666\end{array}$ & 2.3 & 0.9 \\
\hline & p2.16xlarge & 32 & 488 & $20 \mathrm{~Gb}$ & $\begin{array}{l}\text { Intel Xeon E5- } \\
2666\end{array}$ & 2.3 & 7.2 \\
\hline
\end{tabular}

\subsection{Install StarCluster on Client PC}

StarCluster is a utility for creating and managing general purpose computing clusters hosted on Amazon EC2. It minimizes the administrative overhead associated with obtaining, configuring, and managing a traditional computing cluster used in research labs or for general distributed computing applications.

StarCluster is available through the Python Package Index (PyPI). There are three steps to install StarCluster on Linux operation systems (Ubuntu 14.04 as an example) described as following:

(1) Install newest setup tools

\$ sudo apt-get install python-pip

$\$$ sudo pip install -U setuptools

(2) Install dependence packages

\$ sudo apt-get install python-dev, libffi-dev, libssl-dev

(3) Install StarCluster

\$ sudo easy_install Starcluster

\subsection{Build a free virtual cluster on Amazon EC2}

All the informations about how to build and configure a cluster are included in a single StarCluster configuration file. The default configuration file, which could be created by running "starcluster help" at the command-line, lives in 
“ $\sim$.starcluster/config". It is made up of various sections, including three necessary sections [aws info], [keypair], and [cluster], as well as some optional sections [volume], [plugin], [permission] and so on.

There are 5 steps to build and configure a free tier virtual cluster on Amazon EC2.

Step 1: Configure the accounting information of Amazon by modifying the [aws info] section of configuration file.

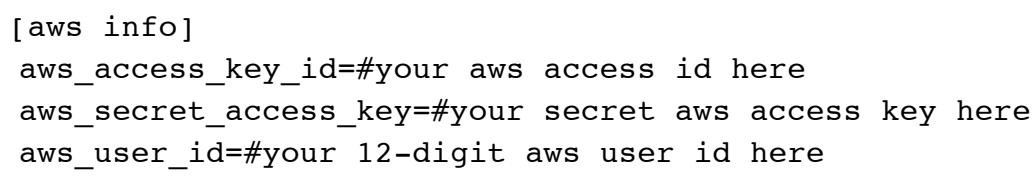

Step 2: Create and configure key.

Create a key file by following shell command.

\$ starcluster createkey mykey -o / /ssh/mykey.rsa

Input information of key by modifying the [key] section in configure file.

[ key mykey]

KEY_LOCATION= /.ssh/mykey $\cdot$ rsa

Step 3: Configure EBS Volume and MPI plugin

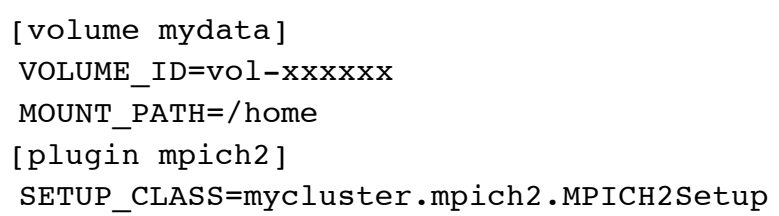

Step 4: Configure the Cluster information.

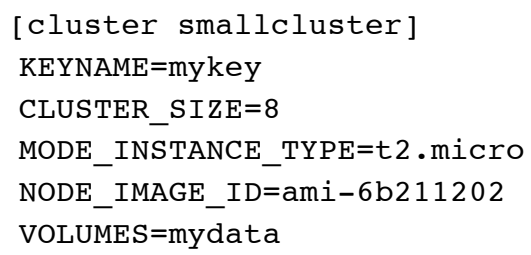

Step 5: Start the cluster.

\$ starcluster start mycluster

The architecture of the virtual cluster built on Amazon EC2 with StarCluster is illustrated in Figure 1. 


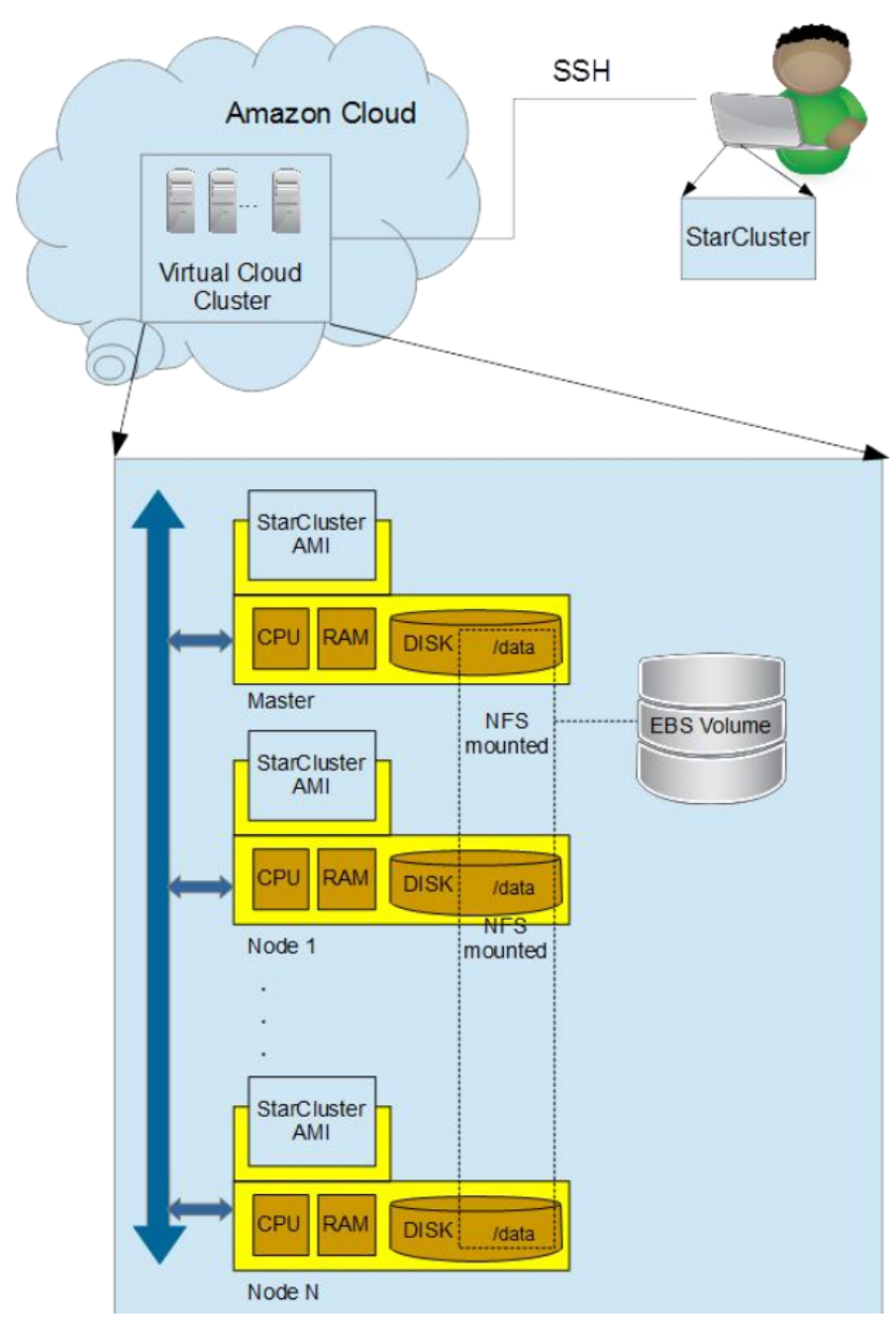

Fig. 1. Architecture of the virtual cluster on Amazon EC2

\section{Evaluating the performance of free virtual cluster}

\subsection{Evaluation methodology}

As mentioned, the goal is to evaluate the performance of the free virtual cluster on Amazon EC2 for scientific computing. In order to achieve this goal, we first measure the raw performance of free tier instance type. Having the raw performance we will be able to predict the performance of a virtual cluster of multiple instances running HPL application Amazon EC2. Then, we evaluate the performance of the free tier virtual cluster. 
The performance metrics for the experiments are based on the critical requirements of scientific applications. We need to evaluate not only the computed performance of the instance in case of running compute intensive applications, but also the performances of memory, network, I/O, which are also important factors on the performance of scientific computing.

Experimental testbed. We use free tier instance (t2.micro) to build a virtual cluster. The configuration of 2 .micro instance is listed in Table 1 . In order to compare the raw performance of $\mathrm{t} 2$.micro instance with commodity $\mathrm{PC}$, we also test the raw performance of a comparing PC, whose configuration is Intel i7 6500u CPU, 8GB DDR III Memory, 250GB SSD hard disk.

Benchmarks. It is important for us to use wide-spread benchmarking tools that have been used in the field of the scientific computing. Currently, there is no single accepted benchmark for scientific computing environments [11]. To deal with this problem, our method both uses traditional benchmarks comprising suites of jobs to be run in isolation and replays workload traces taken from real scientific computing scenario. We design two types of test workloads: Single instance benchmark and Cluster benchmark. These two types workloads all involve executing one or more from a list of four open-source benchmarks: LMbench [12], Bonnie [13], CacheBench [14], and the HPC Challenge Benchmark (HPCC) [15]. The characteristics of these benchmarks are summarized in Table 2.

Table 2. The benchmarks used for cloud performance evaluation

\begin{tabular}{|l|l|l|l|}
\hline \multicolumn{1}{|c|}{ Type } & \multicolumn{1}{c|}{ Suit/Benchmark } & \multicolumn{1}{c|}{ Resource } & \multicolumn{1}{c|}{ Unit } \\
\hline \multirow{3}{*}{$\begin{array}{l}\text { Single Machine Bench- } \\
\text { mark }\end{array}$} & LMbench/all & Many & Many \\
\cline { 2 - 4 } & Bonnie/all & Disk & MBps \\
\cline { 2 - 4 } & CacheBench/all & Memory & MBps \\
\hline \multirow{4}{*}{ Cluster Benchmark } & HPCC/HPL & CPU, float & GFLOPS \\
\cline { 2 - 4 } & HPCC/STREAM & Memory & GBps \\
\cline { 2 - 4 } & HPCC/Random Access & Network & MUPS \\
\hline
\end{tabular}

\subsection{Single-Machine Benchmarks}

In this set of experiments we measure the raw performance of the CPU, I/O, and memory hierarchy of free tier instance and comparing PC using the Single Instance Benchmarks listed in Table 2.

Computation performance. We assess the computational performance of free tier instance (t2.micro) and a comparing PC using the LMbench suite. The performance of float and double operations are illustrated in Figure 2. The results show that the raw performance of free tier instance is equivalent to the comparing PC, which means the free tier instance is not too weak to scientific computing. Furthermore, the float and double performance of free tier instance, arguably the most important for scientific computing, is mixed: excellent addition and bogo but poor div and multiplication capabilities. 


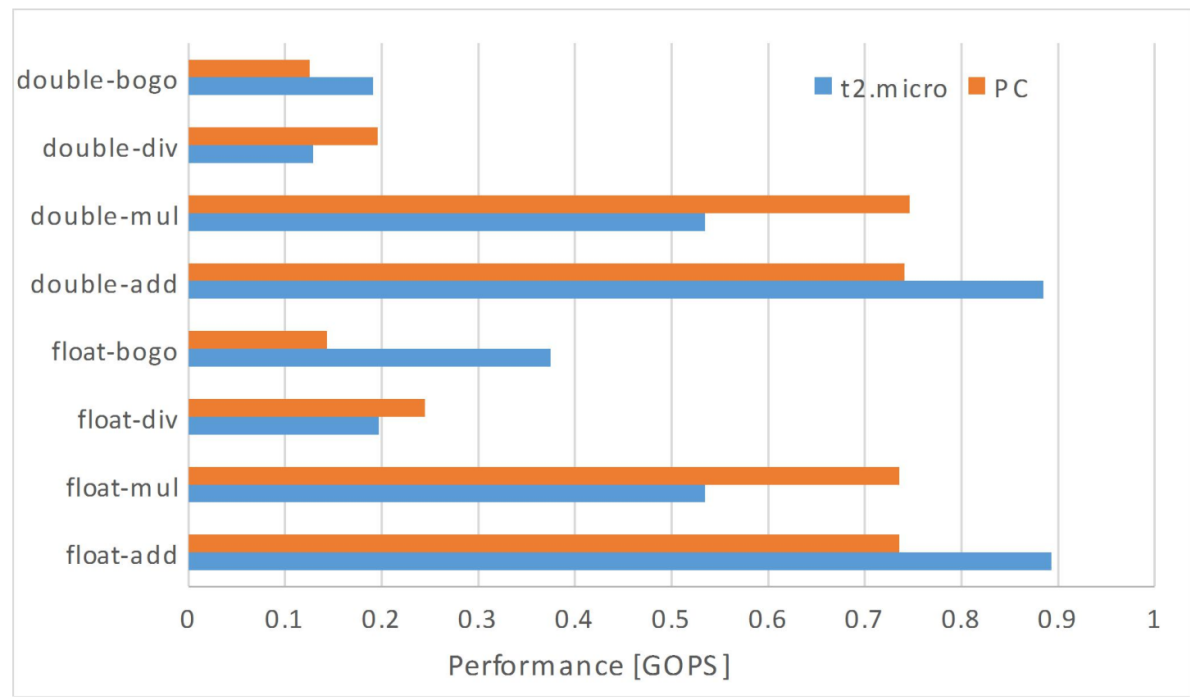

Fig. 2. LMbench results of the performance of $t 2$.micro instance and $\mathrm{PC}$

I/O performance. We assess the I/O performance of free tier instance with Bonnie benchmarks in two steps. The first step is to determine the smallest file size that invalidates the memory cached $\mathrm{I} / \mathrm{O}$, by running the Bonnie suite for eleven file sizes from $1 \mathrm{MB}$ to $2000 \mathrm{MB}$. The result of the rewrite with sequential output benchmark, which involves sequences of readseek- write operations of data blocks that are dirtied before writing, is plotted in Figure 3. From Figure 3 we can see that a performance drop begins with the $50 \mathrm{MB}$ test file and ends at $500 \mathrm{MB}$, which indicates a capacity of the memory-based disk cache of $1000 \mathrm{MB}$ (twice of $500 \mathrm{MB}$ ). Thus, the results obtained for the file sizes above $1000 \mathrm{MB}$ correspond to the real I/O performance of the system, while lower file sizes would be served by the system with a combination of memory and disk operations.

In the second step, we analyze the I/O performance obtained for file size above $1000 \mathrm{MB}$, and summarize the results in Table 3. We also summarize the result of t2.micro with SSD volume and PC. The results show that the I/O performance of free tier instance is much lower than comparing PC. However, we could upgrade the HD volume to the SSD volume, which has the comparable I/O performance with comparing PC. The price for the SSD volume is $\backslash \$ 0.1$ per GB-month, which is affordable for most scientific researchers.

Memory Hierarchy performance. We test the performance of the memory hierarchy using CacheBench on free tier instance and PC, and plot the result in Figure 4 . We find the memory hierarchy size by extracting the major performance drop-offs of Rd-Mod-Wr (rmw) benchmark. The L1/L2 memory sizes are 32 $\mathrm{KB} / 128 \mathrm{~KB}$. We speculate on the existence of a throttling mechanism installed by Amazon to limit resource consumption. If this is true, the performance of computing applications would be severely limited when the working set is near or larger than the L2 size. 
Paper-Build and Evaluate a Free Virtual Cluster on Amazon Elastic Compute Cloud for Scientific Com...

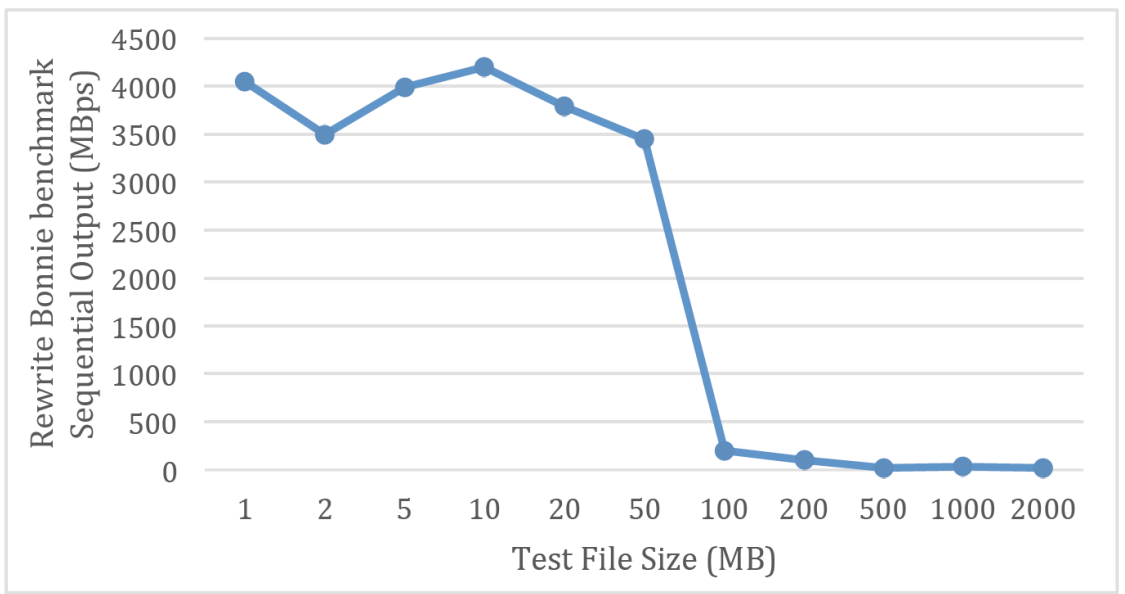

Fig. 3. Bennie rewrite benchmark result. The performance drop indicate the capacity of memory-based disk cache

Table 3. The I/O performance of Amazon EC2 free tier Instance (t2.micro), t2.micro with SSD and comparing PC

\begin{tabular}{|c|c|c|c|c|c|c|}
\hline \multirow[t]{2}{*}{ Platform } & \multicolumn{3}{|c|}{ Seq. Output } & \multicolumn{2}{|c|}{ Seq. Input } & \multirow{2}{*}{$\begin{array}{c}\text { Rand. } \\
\text { Input } \\
\text { [seek/s] }\end{array}$} \\
\hline & $\begin{array}{c}\text { Char } \\
{[M B / s]}\end{array}$ & $\begin{array}{c}\text { Block } \\
{[M B / s]}\end{array}$ & $\begin{array}{c}\text { Rewrite } \\
{[M B / s]}\end{array}$ & $\begin{array}{c}\text { Char } \\
{[M B / s]}\end{array}$ & $\begin{array}{c}\text { Block } \\
{[M B / s]}\end{array}$ & \\
\hline t2.micro & 18.8 & 17.7 & 13.6 & 42.6 & 63.2 & 16.9 \\
\hline t2.micro with SSD & 63.5 & 63.5 & 61.7 & 62.6 & 62.5 & 505.3 \\
\hline Comparing PC & 98.2 & 86.5 & 59.7 & 97.6 & 165.6 & 178.5 \\
\hline
\end{tabular}

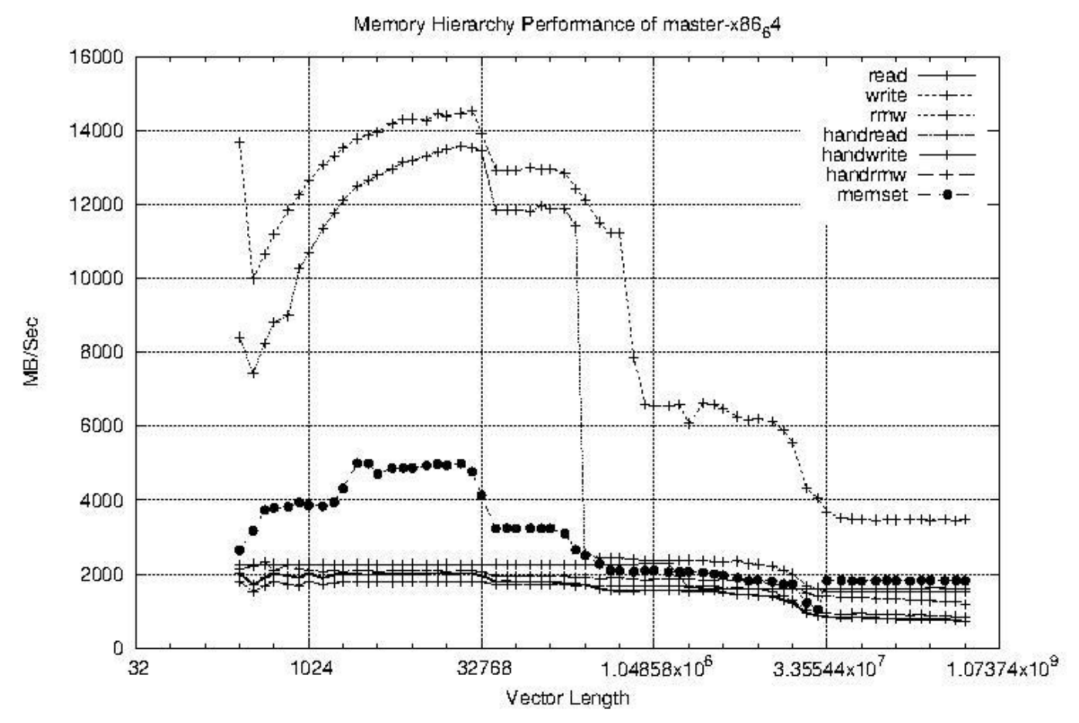

Fig. 4. CacheBench benchmark results of free tier instance (t2.micro) 


\subsection{Multi-Machine Benchmarks}

HPL performance. The performance achieved for the HPL benchmark on the virtual clusters based on free tier instance is plotted in Figure 5. The cluster with one node is able to achieve the performance of 8.447 GFLOPS. For 16 nodes (instances), the performance of cluster achieve 94.37 GFLOPS, which is sufficient for most basic scientific computing.

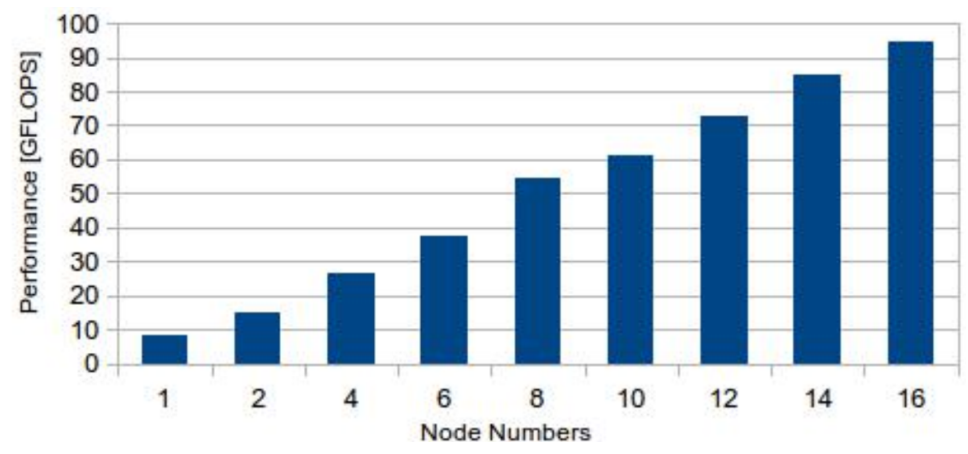

Fig. 5. The HPL (LINPACK) performance of free (t2.micro-based) virtual clusters

To investigate the scalability of the virtual cluster, we calculate the HPL performance speedup and speedup efficiency defined by equations (1)(2), and draw the result in the Figure 6.

$$
\text { speedup }_{N_{-} \text {nodes }}=\frac{H P L \text { performance of } N_{-} \text {nodes }}{\text { HPL performance of one_node }}
$$

$$
\text { Speedup efficiency } y_{N_{-} \text {nodes }}=\frac{\text { Speedup }_{N_{\_} \text {nodes }}}{N}
$$

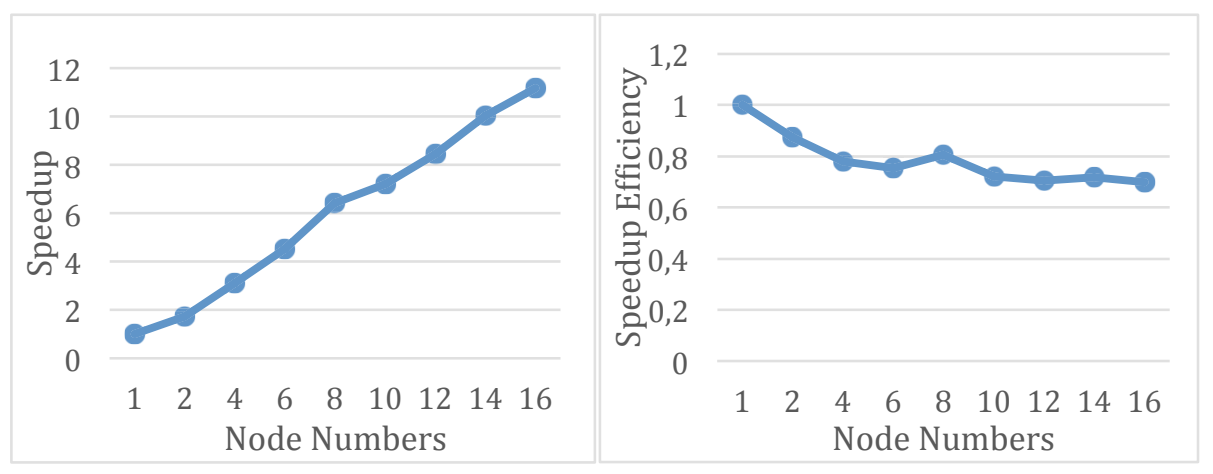

Fig. 6. The speedup and speedup efficiency of HPL (LINPAK) performance of free (t2.microbased) virtual clusters 
From Figure 6 we can see that the speedup increases almost linear and the speedup efficiency is decrease slowly as the number of nodes growing. The speedup efficiency is as higher as the $69.8 \%$ for the 16 nodes (instance) virtual cluster. The results indicate that we can build a virtual cluster on Amazon EC2 with a perfect scalability.

(2) HPCC performance

To obtain the performance of virtual cluster on Amazon EC2, we run the HPCC benchmarks on virtual cluster with different nodes. Table 4 summarizes the obtained results and results published by HPCC for similar size HPC clusters [11]. The performances for the HPL, STREAM, and RandomAccess of the free virtual cluster are better than the published HPCC result, which means the virtual cluster is suitable for the basic scientific computing which has the characteristics of heavy computing. However, due to the poor capacity of the network (listed in Table 1), the virtual cluster has much higher latency which has an important negative impact on some scientific computing exchanging data between different nodes frequently. For these heavy data transfer applications, scientific researcher could develop and test their prototype system of scientific computing on the free virtual cluster first, and move to a higher performance virtual cluster when necessary by choosing more powerful instance type listed in Table 1.

Table 4. The HPCC performance of free cluster on Amazon EC2 and other similar size platforms

\begin{tabular}{|c|c|c|c|c|c|}
\hline $\begin{array}{c}\text { Node Number } \\
\text { /System name }\end{array}$ & $\begin{array}{c}\text { HPL } \\
\text { [GFLOPS] }\end{array}$ & $\begin{array}{c}\text { STREAM } \\
\text { [GBps] }\end{array}$ & $\begin{array}{c}\text { RandomAccess } \\
{[\text { MUPs] }}\end{array}$ & $\begin{array}{c}\text { Latency } \\
{[\boldsymbol{\mu} \text { s] }}\end{array}$ & $\begin{array}{c}\text { Bandwidth } \\
{[\text { GBps] }}\end{array}$ \\
\hline 1 & 8.47 & 5.29 & 24.11 & -- & -- \\
\hline 2 & 14.76 & 4.15 & 24.03 & 171.52 & 0.11 \\
\hline 4 & 26.35 & 4.13 & 24.08 & 131.44 & 0.11 \\
\hline 8 & 54.35 & 4.16 & 23.98 & 138.35 & 0.11 \\
\hline 16 & 94.37 & 4.13 & 24.61 & 173.95 & 0.12 \\
\hline HPCC-228, 8 cores & 27.78 & 2.95 & 10.29 & 5.81 & 0.66 \\
\hline HPCC-227, 16 cores & 55.23 & 2.95 & 10.25 & 6.81 & 0.66 \\
\hline
\end{tabular}

\section{Conclusion}

Scientific computing requires a large number of resources to deliver results for growing problem sizes in reasonable time frame. However, not all scientific researchers have an access of computing resources. Amazon provides a free tier account for cloud computing currently which could be used to build a virtual cluster. Thus, in this paper, we seek to answer two important research questions:"How to build a free tier virtual cluster on Amazon EC2 easily?", and "Is the performance of the free tier virtual cluster base on Amazon EC2 sufficient for scientific computing?". To this end, we first describe how to build a free tier virtual cluster on Amazon with the tool of StarCluster. Then, we perform a comprehensive performance evaluation of the virtual cluster we built before. Our main finding is that the performance of the free virtual cluster on Amazon EC2 is acceptable. Although it is insufficient for scientific compu- 
ting at large, it still appeals the scientific researchers who need computation resources immediately and temporarily. The free virtual cluster built on Amazon EC2 is a suitable for the scientific researcher to develop and test their prototype system of scientific computing without paying anything. They can move to a higher performance virtual cluster when necessary by choosing more powerful instance type on Amazon EC2.

\section{$5 \quad$ References}

[1] Galante G, Bona L.C.E.D, Mury A.R. et al., (2016). "Utilizing Skype for providing learning support for Indonesian distance learning students: An analysis of public clouds elasticity in the execution of scientific application: a survey”, Journal of Grid Computing, vol.14, no.2, pp.193-216. https://doi.org/10.1007/s10723-016-9361-3

[2] Sadooghi I, Martin J.H. Li T. et al., (2015). "Understanding the performance and potential of cloud computing for scientific applications", IEEE Transactions on Cloud Computing, vol. PP, no. 99, pp.1-13.

[3] Coutinho E.F., Flavio R., Sousa C., et al., (2015). "Elasticity in cloud computing: a survey”, Annals of Telecommunications, vol.70, no.7, pp.289-309. https://doi.org/10.1007/s1 2243-014-0450-7

[4] Gupta A., Kale L.V., Gioachin F., et al., (2013). "The who, what, why and how of high performance computing in the cloud", 2013 IEEE 5th International Conference on Cloud Computing Technology and Science, 2-5 Dec. 2013, Bristol, UK. https://doi.org/10.1109/ CloudCom.2013.47

[5] Amazon EC2, https://aws.amazon.com/ec2/, (Jun. 30, 2017, last accessed).

[6] Yi S, Andrzejak A, and Kondo D, (2012). "Monetary Cost-Aware Checkpointing and Migration on Amazon Cloud Spot Instances", IEEE Trans. on Services, vol.5, no.4, pp.512524. https://doi.org/10.1109/TSC.2011.44

[7] Hwang K, Bai X, Shi Y, et al., (2016). "Cloud performance modeling with benchmark evaluation of elastic scaling strategies", IEEE Transactions on parallel and distributed systems, vol.27, no.1, pp.130-143. https://doi.org/10.1109/TPDS.2015.2398438

[8] Iosup A, Ostermann S, Yigitbasi M N, (2011). "Performance Analysis of Cloud Computing Services for Many-Tasks Scientific Computing”, IEEE Transactions on parallel and distributed systems, vol.22, no.6, pp.931-945. https://doi.org/10.1109/TPDS.2011.66

[9] http://star.mit.edu/cluster/, (Jun. 30, 2017, last accessed).

[10] https://aws.amazon.com/ec2/pricing/on-demand/, (Jun. 30, 2017, last accessed).

[11] Ostermann S, Iosup A, Yigitbasi N, et al., (2010). "A Performance analysis of EC2 Cloud Computing Service for Scientific Computing”, Lecture Notes of the Institute for Computer Science, Vol.34, pp115-131. https://doi.org/10.1007/978-3-642-12636-9 9

[12] McVoy L, Stelin C, "LMbench - tools for performance analysis", http://www.bitmover.com/lmbench/, (Jun. 30, 2017, last accessed).

[13] Bray T, (Jun. 30, 2017, last accessed). "Bonnie", http://www.textuality.com/bonnie/.

[14] Mucci P J, London K S, (1998). "Low level architectural characterization benchmarks for parallel computers",Technical Report UT-CS-98-394, Tennessee.

[15] Luszczek P, Baily D H, Dongarra J, et al., (2006). "S12 - The HPC Challenge (HPCC) benchmark suite", In SC, p.213, ACM, New York, (2006). https://doi.org/10.1145/118845 $\underline{5.1188677}$ 


\section{Acknowledgments}

This work is support by the National Science Foundation of China (No. 61273179, No. 61673006), and Science and Technology Research Project of Education Department of Hubei Province of China (No. D20131206, No. B2016034, No. 20141304).

\section{$7 \quad$ Authors}

Jie Xiong (corresponding author) received his Ph.D. Degree in geophysics and Information Technology from China University of Geosciences in 2012. He was a visiting professor at Department of Computer Science and Engineering, Mississippi State University, USA during 2016. He currently is a associate professor at School of Electrical Information, Yangtze University, China. His research interest inlude cloud computing, scientific visualization, and applied geophysical inversion theory.

Shen-Han Shi is a Master Degree Candidate in Electronics and Information at School of Electrical Information, Yangtze University, China. His research interest inlude cloud computing, scientific visualization, and Web application development.

Song Zhang received his M.S and Ph.D Degree in Computer Science from Brown University in 2000, 2006, respectively. He has been associate professor at Department of Computer Science and Engineering, Mississippi State University, USA. His research interests include scientific visualization, data analysis, medical imaging, and computer graphics.

Article submitted 25 June 2017. Published as resubmitted by the authors 27 July 2017. 